\title{
Características antropométricas e bioquímicas de atletas de futebol
}

\section{Anthropometric and biochemical characteristics of football players}

\author{
Joel Saraiva Ferreira'; Petr Melnikov² \\ ${ }^{1}$ Profissional de Educação Física, Doutor em Saúde e Sociedade. Escola de Saúde Pública de Mato Grosso do Sul - ESP/SES/MS, \\ Instituto de Ensino Superior da Funlec - IESF, Faculdade Unigran Capital. Campo Grande, MS - Brasil. \\ ${ }^{2}$ Médico e Químico, Doutor em Química. Faculdade de Medicina - UFMS. Campo Grande, MS - Brasil. \\ Endereço para correspondência \\ Joel Saraiva Ferreira \\ R. Tapés, 33 - Jardim Leblon \\ Campo Grande/MS - CEP 79092-500 \\ falecomjoel@hotmail.com
}

\section{Resumo}

Objetivo: Coletar características antropométricas e bioquímicas de jogadores de futebol de Campo Grande (MS) para avaliar seu estado de saúde. Métodos: Participaram deste estudo 50 jogadores de futebol do sexo masculino, com média de idade de 28,1 anos. Foram medidos: peso corporal, estatura, percentual de gordura, glicose, triglicérides, colesterol total e suas frações LDL e HDL. O teste de correlação de Spearman foi aplicado para o cálculo estatístico, com valor de $p<0,05$ sendo escolhido como nível significativo. Resultados: Os valores obtidos, considerando média \pm desvio-padrão, foram: peso corporal 22,0 $\pm 1,8 \mathrm{~kg}$; percentual de gordura $10,3 \pm 3,2 \% ; 8,3 \mathrm{ml} / \mathrm{dL}$ para glicose; $76,4 \mathrm{ml} / \mathrm{dL}$ para triglicérides; $155,9 \mathrm{ml} / \mathrm{dL}$ para colesterol total, suas frações com 88,5 ml/dL para LDL e 50,8 ml/ dL para HDL. Conclusão: Para a maioria dos indivíduos envolvidos com a prática regular de futebol, esse exercício sistemático garante características antropométricas e bioquímicas com indicadores favoráveis à boa saúde.

Descritores: Colesterol; Exercício físico; Futebol; Glicose; Triglicérides.

\begin{abstract}
Objective: To evaluate the state of health of a group of football players in Campo Grande, Mato Grosso do Sul state, based on anthropometric and biochemical characteristics collected from them. Methods: There were fifty male participants, with an average age of 21.8 years. The following characteristics were measured: body weight, height, percentage of body fat, glucose and triglyceride levels, total cholesterol and its LDL to HDL ratio. The Spearman correlation test was applied for statistical calculations with a $\mathrm{p}<0.05$ significance level. Results: The averages \pm standard deviations obtained were: body weight, $22.0 \pm 1.8 \mathrm{~kg}$; percentage of body fat, $10.3 \pm 3.2 \% ; 8.3 \mathrm{ml} / \mathrm{dl}$ for glucose levels; $76.4 \mathrm{ml} / \mathrm{dl}$ for triglycerides and $155.9 \mathrm{ml} / \mathrm{dl}$ for total cholesterol, $88.5 \mathrm{mg} / \mathrm{dl}$ corresponding to DLD and $50.8 \mathrm{ml} / \mathrm{dl}$ to HDL. Conclusion: For most of individuals engaged in the regular practice of football, this systematic exercise guarantees anthropometric characteristics and biochemical parameters favoring good health.
\end{abstract}

Key words: Cholesterol; Exercise; Glucose; Soccer; Triglycerides. 


\section{Introdução}

A prática de exercícios físicos é de interesse geral, pois a ideia de utilização desse recurso para a promoção da saúde ganha força entre profissionais de saúde e nos meios de comunicação. Com a divulgação de seus benefícios, a população passou a ter conhecimento de que os exercícios físicos promovem adaptações metabólicas importantes para a prevenção de doenças crônicas, principalmente aquelas relacionadas ao sistema cardiovascular ${ }^{1,2}$.

Nesse caso, o tipo de exercício comumente indicado para a manutenção de valores bioquímicos considerados saudáveis é de intensidade moderada, conhecido como aeróbio ${ }^{3}$. No entanto, há indicações de que uma combinação de exercícios físicos em diferentes intensidades (aeróbio e anaeróbio) também produz efeitos fisiológicos benéficos ${ }^{4}$. As opções para sua realização são variadas, podendo ser atividades realizadas de forma espontânea ou por meio da prática sistematizada de modalidades esportivas individuais e coletivas.

Dentre as modalidades esportivas que agregam esforços físicos com demanda energética aeróbia e anaeróbia estão alguns esportes coletivos, como o futebol ${ }^{5}$. Esse esporte tem enorme popularidade no Brasil inteiro, o que acarreta um número elevado de atletas profissionais da modalidade, disputando diversas competições em nível regional, nacional e internacional. Em todos os níveis de competição há períodos de treinamento constituídos de seções de preparação tática, técnica e física, todas iniciadas cerca de 30 dias antes das disputas de campeonatos. Durante o período das competições esportivas, que dura aproximadamente seis meses, o treinamento se mantém em torno das seções citadas.

A condição física e a performance dos atletas de futebol têm sido objeto de estudos, os quais trazem informações sobre as variáveis que mais influenciam o desempenho na modalidade $^{6}$. Em relação aos parâmetros bioquímicos, supõe-se que devido ao treinamento diário e a estrutura adequada de alimentação, tais variáveis permaneçam nos limites tidos como saudáveis nesses jogadores. Geralmente, os ditos parâmetros têm correlação positiva com medidas antropométricas que, por sua vez, dependem do metabolismo energético.

Contudo, na literatura faltam dados qualitativos no que diz respeito ao quadro quantitativo antropométrico-metabólico. As publicações relacionadas a atletas de futebol tratam mais especificamente de fatores relacionados à constituição de tecido ósseo e aspectos psicológicos da preparação dos jogadores ${ }^{7,8,9}$.

Dessa forma, o objetivo neste estudo foi descrever o perfil de marcadores bioquímicos básicos, tais como glicose, triglicérides e lipoproteínas, em atletas da modalidade de futebol, verificando as correlações entre os ditos parâmetros e dados antropométricos individuais para formar um quadro qualitativo. O trabalho faz parte dos estudos da biologia do exercício e permite avaliar objetivamente o estado de saúde dos atletas nas condições reais.

\section{Métodos}

\section{População investigada}

A população deste estudo é composta por 50 jogadores de futebol, os quais representam dois clubes que regularmente disputam o campeonato estadual de Mato Grosso do Sul. Todos os indivíduos são do sexo masculino, na faixa etária de 18 a 35 anos, o que representa uma das características da condição atlética, que é a boa aptidão física atingida nas primeiras décadas da vida adulta.

Foram escolhidos aqueles que atenderam aos seguintes critérios de preparação:

- O treinamento desportivo tinha a mínima periodicidade de cinco vezes por semana, destinando pelo menos 60 minutos a cada sessão.

- O tempo da interrupção dos exercícios nunca excedeu sete dias consecutivos nos últimos três meses. 


\section{Coleta de dados}

Na sede dos clubes esportivos, os técnicos e os atletas receberam informação sobre os propósitos e procedimentos da pesquisa. Para os jogadores que demonstraram interesse em participar do estudo, foi entregue o Termo de Consentimento Livre e Esclarecido (TCLE). Quando houve concordância com o conteúdo e o documento foi assinado, deu-se prosseguimento ao trabalho.

Os atletas receberam prévia orientação sobre a necessidade de comparecerem na data da avaliação, em jejum de 12 horas, para a realização de medidas antropométricas e coleta da amostra de sangue. Foi estipulada uma vestimenta uniforme e adequada para tais procedimentos.

$\mathrm{Na}$ data estabelecida, realizou-se a tomada das medidas antropométricas dos atletas, as quais incluíram o peso corporal $(\mathrm{kg})$, a estatura $(\mathrm{m})$ e quatro dobras cutâneas $(\mathrm{mm})$, sendo estas: tricipital, suprailíaca, subescapular e da panturrilha.

Em seguida, um técnico em laboratório coletou de cada atleta $10 \mathrm{ml}$ de sangue venoso, sendo esse material armazenado em tubos individuais com etiquetas identificadoras por meio de códigos para manter o anonimato do participante. Foram usados tubos de poliestireno a vácuo, siliconizados. Os tubos foram acondicionados em caixa térmica e, posteriormente, transportados à Seção de Análises Clínicas do Hospital Universitário da Universidade Federal de Mato Grosso do Sul (UFMS). Após a centrifugação em 3000 RPM, obteve-se soro límpido e as amostras foram armazenadas e congeladas a $-25{ }^{\circ} \mathrm{C}$. Foi dada atenção para evitar o uso de amostras com sinais de hemólise. Foram determinados: glicose, triglicérides e colesterol com suas frações.

Durante a coleta, armazenamento, transporte e processamento das amostras de sangue, respeitaram-se todas as normas de biossegurança vigentes. Após ser analisado, o material biológico foi devidamente descartado.

Com os valores do peso corporal e da estatura, calculou-se o Índice de Massa Corporal (IMC), que pode ser definido como: IMC = peso corporal $\div$ estatura $^{2}$. Com os valores das dobras cutâneas, calculou-se o percentual de gordura corporal $(\% \mathrm{G})$ de cada atleta por meio de equação específica para a população brasileira ${ }^{10}$.

\section{Aspectos éticos}

Este projeto de pesquisa recebeu parecer favorável (protocolo $\mathrm{n}^{\circ}$ 1.168) do Comitê de Ética em Pesquisa com Seres Humanos da Universidade Federal de Mato Grosso do Sul (CEP/UFMS), atendendo às exigências éticas e científicas fundamentais das pesquisas envolvendo seres humanos.

\section{Critérios estatísticos utilizados}

Os procedimentos estatísticos, além do cálculo da média e do desvio-padrão, incluíram a análise de correlação do \%G e IMC com outras variáveis bioquímicas escolhidas (glicose, triglicérides e colesterol total), aplicando o teste de correlação de Spearmann. O valor de $p$ adotado foi $\leq 0,05$. Os dados foram processados usando o programa BioEstat 5.0.

\section{Resultados}

Na Tabela 1, são resumidos os dados referentes à idade e variáveis antropométricas do grupo estudado; a Tabela 2 contém as informações sobre os parâmetros bioquímicos e a Tabela 3 apresenta os coeficientes de correlação entre dados antropométricos e bioquímicos.

Tabela 1: Idade e varióveis antropométricas de atletas de futebol de Campo Grande, MS $(\mathrm{n}=50)$

\begin{tabular}{ccccc}
\hline Variável & Média & $\begin{array}{c}\text { Desvio } \\
\text { padrão }\end{array}$ & $\begin{array}{c}\text { Valor } \\
\text { mínimo }\end{array}$ & $\begin{array}{c}\text { Valor } \\
\text { máximo }\end{array}$ \\
\hline $\begin{array}{c}\text { Idade (anos) } \\
\quad 21,8\end{array}$ & 3,5 & 18 & 35 \\
$\begin{array}{c}\text { Peso } \\
\text { corporal }(\mathrm{kg})\end{array}$ & 69,6 & 7,2 & 57,2 & 87,9 \\
Estatura $(\mathrm{m})$ & 1,76 & 0,06 & 1,62 & 1,91 \\
$\mathrm{IMC}\left(\mathrm{kg} / \mathrm{m}^{2}\right)$ & 22,5 & 1,8 & 18,7 & 26,4 \\
$\quad \begin{array}{c}\text { Gordura } \\
\text { corporal }(\%)\end{array}$ & 10,3 & 3,2 & 6,2 & 20,6 \\
\hline
\end{tabular}


Em relação à condição antropométrica, nota-se que os valores médios de IMC $\left(22,5 \mathrm{~kg} / \mathrm{m}^{2}\right)$ e o percentual de gordura corporal $(10,3 \%)$ são compatíveis com a exigência motora da modalidade praticada, a qual é constituída de esforços físicos de média e longa duração, com intensidades moderadas e altas. Sua realização é favorecida para indivíduos que não possuam excesso de peso corporal. No entanto, os valores máximos observados nessas duas variáveis possibilitam a inferência de que, mesmo num grupo de atletas, há a presença de indivíduos que possuem excesso de massa corporal, nesse caso, representada pelo desenvolvimento de tecido adiposo.

Tabela 2: Parâmetros bioquímicos de atletas de futebol de Campo Grande, MS $(\mathrm{n}=50)$

\begin{tabular}{ccccc}
\hline Variável & Média & $\begin{array}{c}\text { Desvio } \\
\text { padrão }\end{array}$ & $\begin{array}{c}\text { Valor } \\
\text { mínimo }\end{array}$ & $\begin{array}{c}\text { Valor } \\
\text { máximo }\end{array}$ \\
\hline $\begin{array}{c}\text { Glicose } \\
(\mathrm{ml} / \mathrm{dL})\end{array}$ & 86,3 & 8,0 & 60 & 104 \\
$\begin{array}{c}\text { Triglicérides } \\
(\mathrm{ml} / \mathrm{dL})\end{array}$ & 76,4 & 28,7 & 32 & 149 \\
$\begin{array}{c}\text { Colesterol } \\
\text { total }(\mathrm{ml} / \mathrm{dL})\end{array}$ & 155,9 & 27,7 & 114 & 219 \\
$\begin{array}{c}\text { Colesterol } \\
\text { LDL }(\mathrm{ml} / \mathrm{dL})\end{array}$ & 88,5 & 25,1 & 52,8 & 150,4 \\
$\begin{array}{c}\text { Colesterol } \\
\mathrm{HDL}(\mathrm{ml} / \mathrm{dL})\end{array}$ & 50,8 & 7,5 & 37 & 73 \\
\hline
\end{tabular}

A análise bioquímica do material coletado mostrou que os dados obtidos são adequados para a saúde humana em adultos jovens, e os valores médios de glicose $(86,3 \mathrm{ml} / \mathrm{dL})$, triglicérides (76,4 ml/dL), colesterol total $(155,9 \mathrm{ml} / \mathrm{dL})$, colesterol LDL (88,5 ml/dL) e colesterol HDL (50,8 ml/ dL) não representam alterações. Essa condição favorável à saúde está vinculada a um estilo de vida saudável e compatível com todas as demais exigências da condição atlética, tais como alimentação e repouso adequados, além de menor consumo de bebidas alcoólicas e tabaco.

A análise da correlação entre os dois índices antropométricos (IMC e \%G) e os principais indicadores bioquímicos, averiguados neste estudo, confirma correlação estatisticamente sig-
Tabela 3: Correlação entre varióveis antropométricas e parâmetros bioquímicos de atletas de futebol de Campo Grande, MS $(\mathrm{n}=50)$

\begin{tabular}{cccc}
\hline Parâmetros & $\begin{array}{c}\text { Glicose } \\
(\mathbf{m l} / \mathbf{d L})\end{array}$ & $\begin{array}{c}\text { Triglicérides } \\
(\mathbf{m l} / \mathbf{d L})\end{array}$ & $\begin{array}{c}\text { Colesterol } \\
\text { total }(\mathrm{ml} / \mathrm{dL})\end{array}$ \\
\hline $\begin{array}{c}\text { Índice de } \\
\text { massa }\end{array}$ & $-0,0979$ & $-0,1420$ & $-0,0236$ \\
$\begin{array}{c}\text { corporal } \\
\left(\mathrm{kg} / \mathrm{m}^{2}\right)\end{array}$ & & & \\
$\begin{array}{c}\text { Percentual } \\
\text { de gordura }\end{array}$ & 0,2243 & 0,1052 & $0,3295 *$ \\
corporal $(\%)$ & & & \\
\hline
\end{tabular}

* Correlação significativa $(p<0,05)$.

nificativa apenas entre o percentual de gordura corporal e a concentração de colesterol total. Tais resultados indicam que ambas as variáveis, concentração plasmática de lipoproteínas e o depósito de tecido adiposo, variam juntas e de forma positiva nos atletas que constituem o grupo estudado.

\section{Discussão}

Apesar de o grupo estudado ser formado por indivíduos em uma faixa etária que varia de 18 a 35 anos, nota-se uma média de idade jovem (21,8 $\pm 3,5$ anos), o que confirma a realidade esportiva, pois reflete o ápice do desenvolvimento do tecido conjuntivo correspondente ao desempenho atlético ${ }^{11}$.

De forma geral, a prática sistematizada de exercícios físicos leva a diferentes alterações orgânicas, as quais incluem mudanças nas medidas antropométricas. Há indicação de que exercícios físicos realizados com diferentes níveis de intensidade representam um importante mecanismo de manutenção dos parâmetros antropométricos favoráveis à saúde ${ }^{12}$. É possível que isso se aplique ao caso dos atletas participantes deste estudo, os quais apresentam valores antropométricos considerados adequados à boa saúde e, conjuntamente, ao desempenho esportivo na modalidade praticada.

No caso específico do futebol, os esforços físicos se alternam em situações aeróbias e ana- 
eróbias $^{5}$, indicando que a modalidade necessita de atletas com características corporais que permitam suportar ambas as opções de demanda energética.

Dessa maneira, é presumível que atletas profissionais de futebol tenham uma proporção adequada entre peso corporal e estatura. No caso deste estudo, os valores médios de peso corporal $(69,6 \pm 7,2 \mathrm{~kg})$ e de estatura $(1,76 \pm 0,06$ $\mathrm{m})$ demonstram essa proporção por meio do valor médio do IMC, que foi $22,5 \mathrm{~kg} / \mathrm{m}^{2}$, considerado como apropriado para os padrões de saúde $\mathrm{e}^{13}$ e também para a performance atlética ${ }^{14}$.

O percentual de gordura corporal é outra variável relacionada à constituição corpórea de atletas com fundamental importância para a classificação do esportista saudável e com bom desempenho; descreve o conteúdo corpóreo de quantidade de massa magra e massa gorda. O valor médio dessa variável $(10,3 \pm 3,2 \%)$ nos atletas participantes do estudo encontra-se dentro de níveis aceitáveis para ambas as condições mencionadas. Esse valor corresponde ao padrão obtido num estudo que analisou atletas da mesma modalidade, que atuam no estado de São Paulo ${ }^{6}$.

Para ambos os índices antropométricos aqui analisados, os dados do grupo indicam a presença de seis indivíduos (12\%) com excesso de massa corporal (IMC $\geq 25 \mathrm{~kg} / \mathrm{m}^{2}$ ). Quatro indivíduos (8\%) têm excesso de tecido adiposo (\%G $\geq 15)$, o que corresponde a valores disponíveis na literatura ${ }^{15}$. Esses resultados podem estar relacionados com sérias alterações do regime nutricional (fast food, carnes gordurosas, etc.).

Em relação aos parâmetros bioquímicos, esses têm sido utilizados em diferentes estudos para acompanhar as condições de saúde associada à prática de exercício físico, com indivíduos de diferentes faixas etárias, gêneros e nível habitual de atividade física ${ }^{16,17}$. Neste estudo, optouse pela verificação da glicemia em jejum, dos triglicérides e das lipoproteínas plasmáticas, tendo em vista a boa aplicabilidade desses testes com o propósito de investigar as condições de adaptação cardiovascular de atletas.
A atual Diretriz Brasileira sobre Dislipidemias $^{18}$ estabelece valores de referência para todos os parâmetros investigados neste estudo e, em todos os casos, o valor médio do grupo pesquisado está equivalente àqueles classificados como favoráveis à saúde. Mesmo assim, a respeito do colesterol total, nota-se que há indivíduos com valores acima daqueles considerados adequados, até superiores a $200 \mathrm{mg}$ / dL. Esses níveis podem ser devidos tanto aos fatores nutricionais como à predisposição genética para a hipercolesterolemia que nem sempre é detectada. Nas demais variáveis bioquímicas, os valores médios e os máximos correspondem aos níveis adequados ao bom funcionamento do sistema cardiovascular.

Por se tratar de um grupo de praticantes de exercícios físicos com intensidade e volume elevados, é possível que os índices obtidos na constituição corpórea, relacionados aos casos de excesso de massa adiposa, sejam normalizados. Logo, com a manutenção da rotina de treinamentos já estabelecida pode ocorrer a redução do nível de colesterol. Conforme os dados da literatura, a diminuição do peso corporal já confirmou ser um mecanismo eficiente para a redução de lipoproteínas plasmáticas. Vários pesquisadores $^{19}$ observaram condições favoráveis à manutenção de parâmetros bioquímicos adequados naqueles indivíduos que realizam exercícios físicos de forma regular e sistemática. O estudo traz uma ampla revisão de pesquisas realizadas na segunda metade do século $X X$, nas quais houve uma diminuição paralela do peso corporal e as gorduras (colesterol e triglicérides).

Os parâmetros bioquímicos e antropométricos escolhidos para este estudo são variáveis diretamente ligadas a uma série de doenças crônicas, principalmente aquelas relacionadas ao sistema cardiovascular ${ }^{1}$. Nesse caso, a correlação significativa entre o \%G e a quantidade de colesterol total no organismo dos atletas indica que, mesmo naqueles indivíduos ativos fisicamente, o excedente de tecido adiposo se correlaciona com o metabolismo de lipoproteínas no organismo. 
Em um estudo com os profissionais de futebol, realizado para o controle de enfermidades cardiovasculares, foram comparados vários fatores de risco referentes aos atletas estudados com a coorte da população em geral. Foi mostrado que alguns fatores prevaleceram no grupo de atletas e entre eles, principalmente, nos atacantes $^{20}$. Tais dados contrastam com os resultados deste estudo, posto que os valores médios dos parâmetros bioquímicos e antropométricos encaixam nos intervalos fisiológicos do sistema cardiovascular.

Contudo, é de alta relevância que, individualmente, os fatores de risco nos atletas sejam monitorados rigorosamente e, nos casos em que haja alteração nesses índices, o esportista e o médico da equipe precisam ser imediatamente informados.

\section{Conclusões}

A prática regular da modalidade esportiva futebol, na maioria dos atletas proporciona características antropométricas e bioquímicas favoráveis à saúde e ao desempenho esportivo.

Em parte dos atletas, observa-se correlação positiva significativa entre o percentual de gordura corporal e a concentração de colesterol total, constituindo fator de risco de doença cardiovascular.

A ocorrência de excesso de massa corporal, nesse caso, representada pelo desenvolvimento de tecido adiposo, pode ser vinculada à ingesta calórica inadequada, já que o gasto energético diário é elevado, em razão da prática esportiva cotidiana do grupo avaliado.

\section{Agradecimentos}

Os autores agradecem o apoio recebido da Coordenação de Aperfeiçoamento de Pessoal de Nível Superior (CAPES) e da Fundação de Apoio ao Desenvolvimento do Ensino, Ciência e Tecnologia de Mato Grosso do Sul (FUNDECT).

\section{Referências}

1. Fogelholm M, Lahti-Koski M. Community healthpromotion interventions with physical activity: does this approach prevent obesity? Scand J Food Nutrition. 2002;46(4):173-7.

2. Schaefer EJ. Lipoproteins, nutrition, and heart disease. Am J Clin Nutrition. 2002;75:191-212.

3. Almeida MB, Araújo CGS. Effects of aerobic training on heart rate. Rev Bras Med Esporte. 2003;9(2):113-20.

4. Polito MD, Simão R, Senna GW, Farinatti PTV. Hypotensive effects of resistance exercises performed at different intensities and same work volumes. Rev Bras Med Esporte. 2003;9(2):72-7.

5. Guerra I, Soares EA, Burini RC. Aspectos nutricionais do futebol de competição. Rev Bras Med Esporte. 2001;7:200-6.

6. Prado WL, Botero JP, Guerra RLF, Rodrigues CL, Cuvello LC, Dâmaso AR. Perfil antropométrico e ingestão de macronutrientes em atletas profissionais brasileiros de futebol, de acordo com suas posições. Rev Bras Med Esporte. 2006;12(2):61-5.

7. Corrêa DKA, Alchiri JC, Duarte LRS, Strey MN. Excelência na produtividade: a performance dos jogadores de futebol profissionais. Psicologia: Reflexão e Crítica. 2002;15(2):447-60.

8. Laabes AP; Vanderjagt DJ; Obadofin MO; Sendeht AJ; Glew RH. Assessment of the bone quality of black male athletes using calcaneal ultrasound: a cross-sectional study. Nutr Metab. 2008;5(13):1-8.

9. Ruffing JA; Cosman F; Zion M; Tendy S; Garrett P; Lindsay R; Nieves JW. Determinants of bone mass and bone size in a large cohort of physically active young adult men. Nutr Metab. 2006;3(14):1-10.

10. Petroski EL, Pires-Neto CS. Validação de equações antropométricas para a estimativa da densidade corporal em homens. Rev Bras Atividade Física e Saúde. 1996;1(3):5-14.

11. Tripton KD, Wolfe RR. Exercise, protein metabolism and muscle growth. Int J Sport Nutri Exerc Metab. 2001;11:109-32.

12. Moreira MM, Souza HPC, Schwingel PA, Couto de Sá CK, Zoppi CC. Efeitos do exercício aeróbico e anaeróbico em variáveis de risco cardíaco em adultos com sobrepeso. Arq Bras Cardiol. 2008;91(4):219-26. 
13. World Health Organization - WHO. Preventing and managing the global epidemic of obesity. Report of the World Health Organization Consultation of Obesity. Geneva: WHO;1997.

14. Reylly T, Bangsbo J, Franks A. Anthropometric and physiological predispositions for elite soccer. J Sports Sci. 2000;18:669-83.

15. Heyward VH, Stolarczik LM. Applied body composition assessment. Champaign: Human Kinetics; 1996.

16. Guedes DP, Guedes JEP, Barbosa DS, Oliveira JA. Dispêndio energético diário e níveis de lipídeoslipoproteínas plasmáticos em adolescentes. Rev Bras Med Esporte. 2007;13(2):123-8.

17. Teixeira M, Kasinski N, Izar MCO, Barbosa LA, Novazzi JP, Pinto LA, Tufic S, Leite TF, Fonseca FAH. Efeitos do exercício agudo na lipemia pósprandial em homens sedentários. Arq Bras Cardiol. 2006;87(1):3-11.
18. Diretriz Brasileira Sobre Dislipidemias e Prevenção da Aterosclerose (IV). Sociedade Brasileira de Cardiologia. Arq Bras Cardiol. 2007;88(Supl I):S1-8.

19. Datillo AM, Kris-Etherton PM. Effects of weight reduction on blood lipids and lipoproteins: a metaanalysis. Am J Clin Nutr. 1992;56(2):320-8.

20. Selden MA, Helzberg JH, Waeckerle JF, Browne JE, Breder JH, Monaco ME, Tanq F, O'Keefe JH. Cardiometabolic abnormalities in current National Football League players. Am J Cardiol. 2009;103(7):969-71. 